VOL. 76 (2007) [133-136]

\title{
ON THE INTEGERS REPRESENTED BY $x^{4}-y^{4}$
}

\author{
ANDRZEJ DĄBROWSKI
}

\begin{abstract}
Let $p$ be a prime number $\geqslant 5$, and $n$ a positive integer $>1$. This note is concerned with the diophantine equation $x^{4}-y^{4}=n z^{p}$. We prove that, under certain conditions on $n$, this equation has no non-trivial solution in $\mathbf{Z}$ if $p \geqslant C(n)$, where $C(n)$ is an effective constant.
\end{abstract}

\section{INTRODUCTION}

By the work of Hellegouarch, Frey, Serre, Ribet, Wiles, Taylor and many others, we can reduce the study of a class of ternary diophantine equations (generalised Fermat equations) to modern techniques coming from Galois representations and modular forms. In all known cases, the proofs follow a variant of the method of Frey curves and Ribet's level-lowering theorem.

Let $n$ be a positive integer $>1$, and $p$ an odd prime, $\operatorname{gcd}(n, p)=1$. Let $v_{l}(n)$ be the exact power of $l$ dividing $n$, and let $\alpha=v_{2}(n)$. Consider the equation

$$
x^{4}-y^{4}=n z^{p}, \quad \operatorname{gcd}(x, y)=1 .
$$

Let $N=2^{4} r^{\prime}(n)$, where $r^{\prime}(n)$ denotes the product of odd prime divisors of $n$. Let $g_{0}^{+}(N)$ denote the dimension of the C-vector space of newforms of weight 2 with respect to the congruence subgroup $\Gamma_{0}(N)$. Let $\mu(N)$ be the index of $\Gamma_{0}(N)$ in $S L(2, \mathrm{Z})$. Put

$$
F(N):=\left(\sqrt{\frac{\mu(N)}{6}}+1\right)^{2 g_{0}^{+}(N)}
$$

Darmon [1] showed that, for a prime number $p \geqslant 11$, the equation $x^{4}-y^{4}=z^{p}$ has no non-trivial solution if $p \equiv 1(\bmod 4)$ or $z$ is even. We combine the methods of Darmon [1] and Kraus [4] to prove the following general result.

Received 11th December, 2006

The article was written during my visit to the Max-Planck-Institut für Mathematik in Bonn in September-December 2006. I would like to thank the Institute for the hospitality and support.

Copyright Clearance Centre, Inc. Serial-fee code: 0004-9727/07 \$A2.00+0.00. 


\section{THEOREM 1.}

(i) Let $\alpha \geqslant 1$, and let $p$ be a prime $\geqslant 5$. Then the equation

$$
x^{4}-y^{4}=2^{\alpha} z^{p}, \quad \operatorname{gcd}(x, y)=1
$$

has no non-trivial solution in integers.

(ii) Let $\alpha \geqslant 1$ and let $p$ be a prime $>\max (F(N), 3)$. Assume that $p \nmid n$ and $v_{l}(n)<p$ for any prime $l$. Assume that there is no elliptic curve over $\mathbf{Q}$ of conductor $N$, with all its 2-division points defined over $\mathbf{Q}$. Then (1) has no non-trivial solution in integers.

(iii) Let $\alpha=0$ and let $p$ be a prime $>\max (F(N), F(2 N), 3)$. Assume that $p \nmid n$ and $v_{l}(n)<p$ for any prime $l$. Assume that there is no elliptic curve over $\mathbf{Q}$ of conductor $N$, with all its 2-division points defined over $\mathbf{Q}$. Then (1) has no non-trivial solution in integers.

Let $E$ be an elliptic curve over $\mathbf{Q}$, of conductor $2^{k} q, q$ an odd prime. If $E$ has all its 2-division points defined over $\mathbf{Q}$, then $q$ is a Fermat or a Mersenne prime ([3], or [4, Lemma 6]). Using the arguments in ([4, p. 1162]), we obtain

Corollary 1. Let $q$ be an odd prime, not of the type $2^{m} \pm 1$, satisfying $p>(\sqrt{8 q+8}+1)^{2 q-2}$. Let $\alpha \geqslant 0, \beta>0$ be integers. Then the equation $x^{4}-y^{4}=2^{\alpha} q^{\beta} z^{p}$ has no non-trivial solution in integers.

\section{Proof of TheOREM 1}

Let $a^{4}-b^{4}=n c^{p}$ be a solution to equation (1). Let

$$
E: y^{2}=x^{3}+4 a b x^{2}-\left(a^{2}-b^{2}\right)^{2} x
$$

denote the corresponding Frey curve (compare [1]). We have

$$
c_{4}=2^{4}\left[2^{4} a^{2} b^{2}+3\left(a^{2}-b^{2}\right)^{2}\right], c_{6}=-2^{7}\left[2^{5} a^{2} b^{2}+3^{2}\left(a^{2}-b^{2}\right)^{2}\right]
$$

and $\Delta=2^{6} n^{2}\left(a^{2}-b^{2}\right)^{2} c^{2 p}$. Let $\Delta_{E}$ and $N_{E}$ denote the minimal discriminant and conductor of $E$, respectively.

LEMMA 1.

(i) If $\alpha=0$ and $c$ is odd, then $\Delta_{E}=2^{6} n^{2}\left(a^{2}-b^{2}\right)^{2} c^{2 p}$ and $N_{E}=2^{5} r^{\prime}(n c)$.

(ii) If $\alpha \geqslant 1$ or $c$ is even, then $\Delta_{E}=2^{-6} n^{2}\left(a^{2}-b^{2}\right)^{2} c^{2 p}$ and $N_{E}=2^{4} r^{\prime}(n c)$.

Proof: (i) In this case a model (3) is global minimal. The curve has multiplicative reduction at any odd prime $r$ dividing $\Delta_{E}$, since $v_{r}\left(c_{4}\right)=0$. On the other hand, 
$v_{2}\left(c_{4}\right)=4, v_{2}\left(c_{6}\right)=7$ and $v_{2}\left(\Delta_{E}\right)=6$, hence using [8], Table IV, we obtain $v_{2}(N)=5$. (ii) In this case, the model

$$
y^{2}=x^{3}+a b x^{2}-2^{-4}\left(a^{2}-b^{2}\right)^{2} x .
$$

is global minimal. Here we have $v_{2}\left(c_{4}\right)=4, v_{2}\left(c_{6}\right)=6$ and $v_{2}\left(\Delta_{E}\right) \geqslant 12$, hence using again $\left[8\right.$, Table IV], we obtain $v_{2}(N)=4$.

Let

$$
\rho: \operatorname{Gal}(\overline{\mathbf{Q}} / \mathbf{Q}) \rightarrow \operatorname{Aut}(E[p]) \simeq G L_{2}\left(\mathbf{F}_{p}\right)
$$

be the Galois representation associated to the $p$-division points of $E$.

Lemma 2. Assume $p \geqslant 5$. Then $\rho$ is absolutely irreducible.

Proof: $E$ has all its 2-division points defined over $\mathbf{Q}$, hence the result follows from [6, Theorem 3] (If $\rho$ is reducible, then we are in case (iii) with $p \leqslant 3$.); see also [7, Theorem 1.3].

Let $N(\rho)$ denote the Artin conductor of $\rho$, as defined in [10].

LEMMA 3. Let $p$ be an odd prime, $\operatorname{gcd}(n, p)=1$. We have $N(\rho)=2^{k} r^{\prime}(n)$, where $k=5$ if $\alpha=0$ or $c$ is odd, and $k=4$ if $\alpha \geqslant 1$ or $c$ is even.

PROof: $E$ has additive reduction at 2, hence $v_{2}(N(\rho))=v_{2}\left(N_{E}\right)$ (see [5]). Now use Lemma 1, and the properties of $N(\rho)([\mathbf{1 0}$, p. 191]).

Elliptic curves $E$ defined by (3) are semistable at 3 and 5 , hence modular due to the work of Wiles [11] and Diamond [2]. Applying the "lowering the level" result of Ribet [9] we conclude that $\rho$ arises from a cuspidal newform of weight 2 and level $2^{k} r^{\prime}(n)$.

COMPLETION OF THE PROOF OF THEOREM 1. (i) The space of cuspidal newforms of weight 2 with respect to $\Gamma_{0}(16)$ is empty, hence the assertion follows. Proofs of (ii) and (iii) follow the same line as the proof of $[4$, Theorem 1]. We omit the details.

\section{REFERENCES}

[1] H. Darmon, 'The equation $x^{4}-y^{4}=z^{p}$ ', C. R. Math. Rep. Acad. Sci. Canada 15 (1993), 286-290.

[2] F. Diamond, 'On deformation rings and Hecke rings', Ann. of Math. 144 (1996), 137-166.

[3] W. Ivorra, 'Courbes elliptiques sur $\mathbf{Q}$, ayant un point d'ordre 2 rationnel sur $Q$, de conducteur $2^{N} p$ ', Dissertationes Math. 429 (2004), 55pp.

[4] A. Kraus, 'Majorations effectives pour l'équation de Fermat généralisée', Canad. J. Math. 49 (1997), 1139-1161.

[5] A. Kraus, 'Détermination du poids et du conducteur associé aux représentations des points de p-torsion d'une courbe elliptique', Dissertationes Math. 364 (1997), 39pp.

[6] B. Mazur, 'Rational isogenies of prime degree', Invent. Math. 44 (1978), 129-162.

[7] L. Merel, 'Arithmetic of elliptic curves and diophantine equations', J. Theor. Nombres Bordeaux 11 (1999), 173-200. 
[8] I. Papadopoulos, 'Sur la classification de Néron des courbes elliptiques en caractéristique résiduelle 2 et 3', J. Number Theory 44 (1993), 119-152.

[9] K. Ribet, 'On modular representations of $\mathrm{Gal}(\overline{\mathbf{Q}} / \mathbf{Q})$ arising from modular forms', Invent. Math. 100 (1990), 431-476.

[10] J.-P. Serre, 'Sur les représentations modulaires de degré 2 de Gal( $\bar{Q} / \mathbf{Q})$ ', Duke Math. J. 54 (1987), 179-230.

[11] A. Wiles, 'Modular elliptic curves and Fermat's Last Theorem', Ann. of Math. 141 (1995), 443-551.

Institute of Mathematics

University of Szczecin

ul. Wielkpolska 15

70-451 Szczecin

Poland

e-mail: dabrowski@sus.univ.szczecin.pl 